\title{
Ultrastructural Studies on the Chromatin Fibers during Spermiogenesis in the Cockroach, Blattella germanica
}

\author{
Aiko Sakai-Wada and Yukiko Hikita \\ Department of Biology, Faculty of Science, Nara Women's University, \\ Nara, 630 Japan
}

Received June 6, 1983

In the final phase of spermatogenesis, spermiogenesis, the spermatid differentiates into a mature spermatozoa or sperm of a species-specific form, consisting of a head, middle piece and tail. During spermiogenesis, the round nucleus with haploid genomes becomes compact with a round, oval and needle-shaped contour and chromosomes become tightly packed inside the small sperm nucleus.

Biochemical studies have shown that DNA of mature sperm nuclei is inert but that during spermiogenesis, chromosomal basic proteins are rapidly replaced by protamine or arginine-rich protein from somatic histone.

Many workers have studied the fine structure of nuclei during spermiogenesis. However, there have been few electron microscopic cytochemical studies on the replacement of nuclear protein (Courtens and Loir 1981b), or on the fine structure of elementary fibers of chromatin of about $30 \mathrm{~nm}$ width ( $30 \mathrm{~nm}$-chromatin fiber) during replacement by protamine or arginine-rich histone.

In the present work the process of replacement of nuclear basic protein of somatic histone by arginine-rich protein, was examined in relation to the morphological changes of $30 \mathrm{~nm}$-chromatin fibers in the nucleus during spermiogenesis of the cockroach, Blattella germanica.

\section{Material and methods}

The testis of Blattella germanica was used.

1) Conventional fixation method

Materials were fixed in a mixture of $5 \%$ glutaraldehyde and $4 \%$ paraformaldehyde adjusted to $\mathrm{pH} 7.0$ with phosphate buffer, for 3 hours at $4{ }^{\circ} \mathrm{C}$. Then they were rinsed with the same buffer solution and post-fixed in $1 \% \mathrm{OsO}_{4}$ adjusted to $\mathrm{pH} 7.0$ with the same buffer. They were then dehydrated in a graded series of acetone and embedded in epoxy resin. Ultrathin sections were cut, stained with lead citrate and examined in an electron microscope.

2) PTA-staining

Materials were fixed with aldehyde as in the conventional procedure for 3 hours at $4^{\circ} \mathrm{C}$. Then they were rinsed with the same buffer, promptly dehydrated in a graded series of acetone, and immersed in $1 \%$ phosphotungstic acid (PTA) in absolute acetone overnight in a refrigerator. They were then dehydrated in acetone 


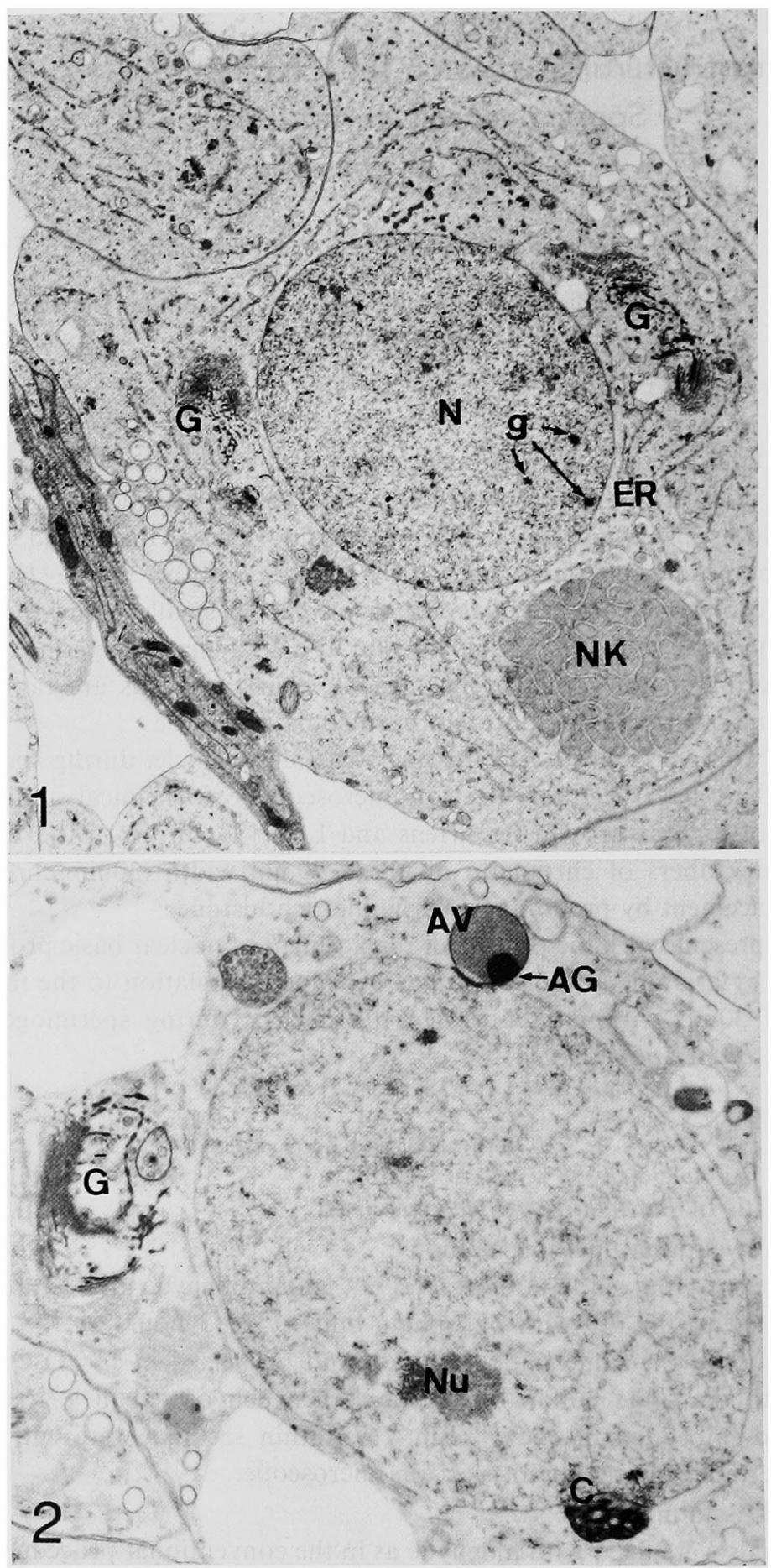



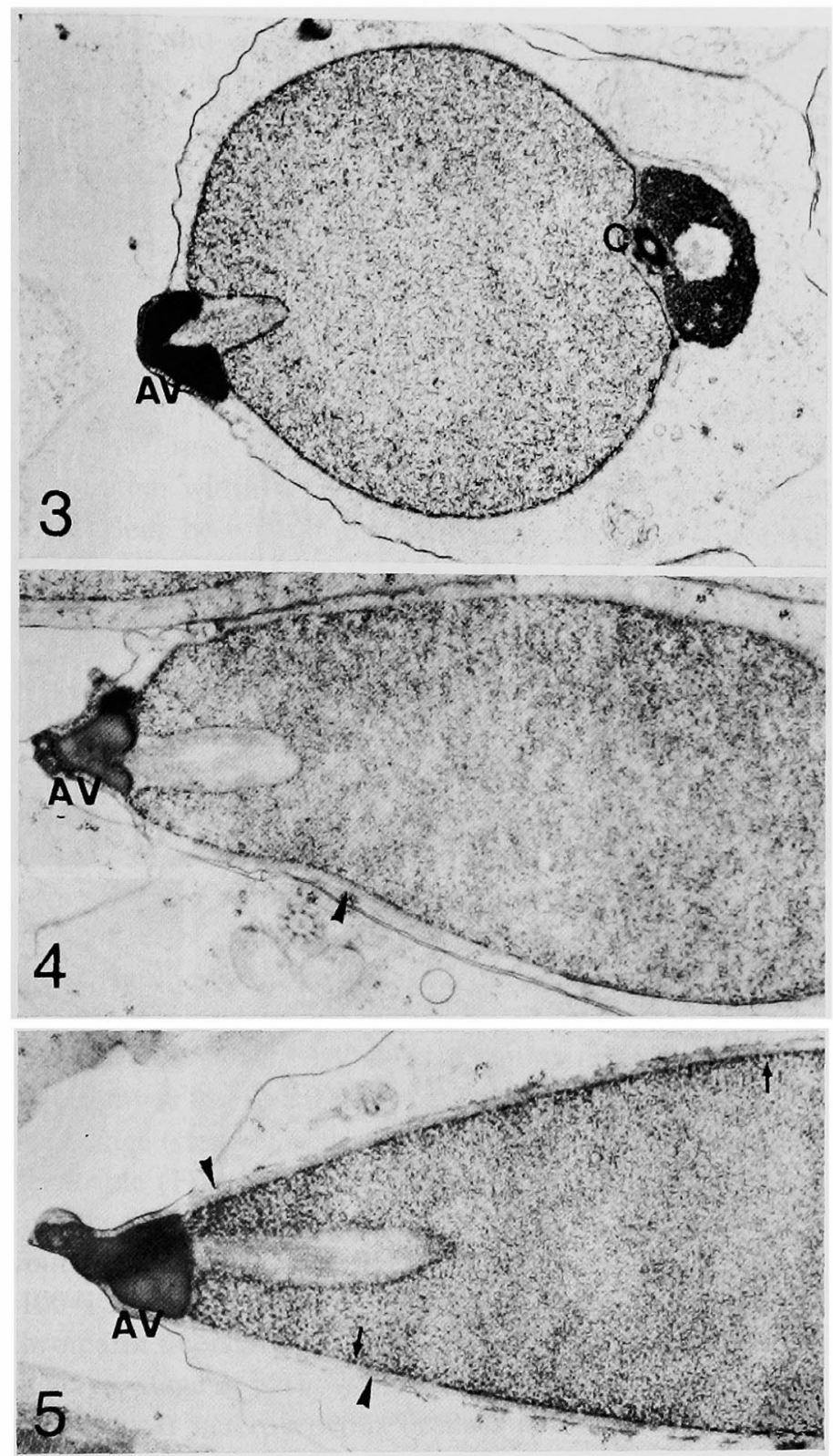

Abbreviations: AV, acrosomal vesicle; AG, acrosomal granule; C, centriole; ER, endoplasmic reticulum; G, Golgi bodies; g, small granules of $100-120 \mathrm{~nm}$ width; N, nucleus; NK, nebenkern; $\mathrm{Nu}$, nucleolus. Arrow heads in all figures show microtubules.

Figs. 1-9, were obtained by conventional fixation. 1, spermatid cell in the early stage of spermiogenesis, soon after meiotic division (stage 1). $\times 7000 . \quad 2$, cell in stage 2 . The acrosomal vesicle and centriole adhere to the surface of the nucleus. $\times 10000$. 3, cell in stage 3 . The nucleus is round. $\times 15000$. 4 , oval nucleus in stage $4 . \times 15000$. 5 , anterior part of a spindle-shaped nucleus in stage $5 . \times 15000$. 


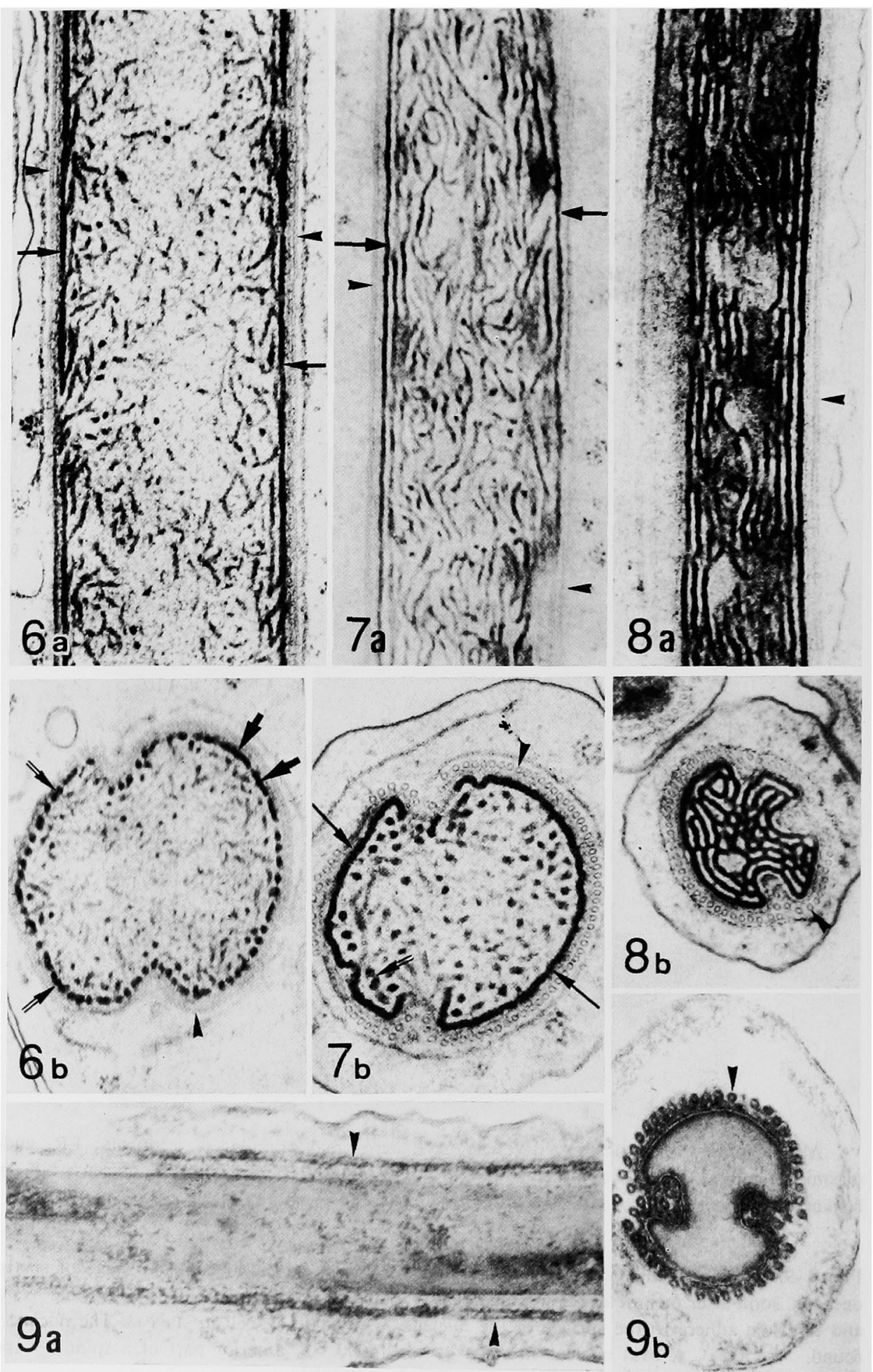


and propylene oxide and embedded in epoxy resin. Ultrathin sections were made and examined without staining in an electron microscope, HU-12A.

\section{Results}

In spermatids of $B$. germanica just after meiotic division, the nucleus is round and $6.5 \mu \mathrm{m}$ in diameter. During spermiogenesis, the nucleus becomes oval, spindle-shaped, rod-shaped and finally needle-shaped and about $0.25 \mu \mathrm{m}$ wide and $50 \mu \mathrm{m}$ long. In parallel with this change in nuclear shape, chromatin fibers in the nucleus, which are about $25 \mathrm{~nm}$ wide in early stages of spermiogenesis, change from a wavy to a straight form. In very late stages of spermiogenesis, the straight chromatin fibers of $30 \mathrm{~nm}$ width become tightly packed side by side, and the mature spermatozoan nucleus becomes a mass of tightly condensed chromatin, looking as if it was in a crystalline state. In the present studies sperm maturation of $B$. germanica was divided into nine stages on the basis of the appearance of the nucleus and the chromatin fibers. The figures obtained by conventional fixation were used as standards for comparison with those obtained by the PTA-staining method.

Figs. 1-9 were obtained by the conventional fixation procedure.

The first stage is soon after meiotic division. At this stage, the nucleus is round and 6-8 $\mu \mathrm{m}$ in diameter (Fig. 1, N), and is filled with wavy chromatin fibers of $10-25 \mathrm{~nm}$ width. Some electron-dense granules of chromatin of 100-120 nm diameter are seen scattered among the chromatin fibers (Fig. 1, g). The nucleolus is recognized as a cluster of electron dense threads and granules of about 100-200 nm diameter (Fig. 2, Nu). In the cytoplasm, the nebenkern, or aggregates of mitochondria (Fig. 1, NK), large sized Golgi bodies, which take part in organization of acrosomal vesicles (Fig. 1, G. Sakai-Wada and Kuriyama 1980), and a fine endoplasmic reticulum (Fig. 1, ER) are observed.

The second stage (stage 2) is shown in Fig. 2. A round acrosomal vesicle (Fig. 2, AV) and centriole (Fig. 2, C) which becomes the basal body of the sperm tail, are both seen adhering to the surface of the round nucleus. The nucleus contains evenly distributed wavy chromatin fibers of $10-25 \mathrm{~nm}$ width. The electron dense granules of $100-120 \mathrm{~nm}$ diameter seen in the nucleus in stage 1 are reduced in number. Chromatin fibers are distributed evenly throughout the nucleus.

The next stage, stage 3, is shown in Fig. 3. In this stage, the nucleus is round, but, its contents appear heterogeneous because two kinds of chromatin fibers with

Figs. 6-9. 6a, part of a nucleus in stage 6 sectioned longitudinally. Arrows indicate a straight chromatin fibers of $30 \mathrm{~nm}$ width. $\times 30000,6 \mathrm{~b}$, cross section of a nucleus in stage 6 . Double arrows show $30 \mathrm{~nm}$-chromatin fibers adhering to each other. Thick arrows indicate a sheet of chromatin suggested to be formed by side-to-side fusion of chromatin fibers of $30 \mathrm{~nm}$ width. $\times 30000$. 7a, part of a longitudinal section of a nucleus in stage 7. Arrows indicate the straight chromatin fibers of $30 \mathrm{~nm}$ width. $\times 30000.7 \mathrm{~b}$. cross section of a nucleus at stage 7 . Arrows show a sheet of chromatin. $\times 30000 . \quad 8 \mathrm{a}$, part of a longitudinal section of a nucleus at stage 8 . Chromatin is seen as parallel stripes. $\times 40000.8 \mathrm{~b}$, transverse section of a nucleus at stage 8 . Chromatin appears as a network. $\times 40000.9 \mathrm{a}$, part of a longitudinal section of a nucleus at stage 9. The nucleus is grey with scattered electron-dense grains. $\times 60000$. 9b, cross section of a nucleus at stage $9 . \quad \times 60000$. 
different diameters become localized in different regions: fibers of $25 \mathrm{~nm}$ diameter are seen in the periphery, and those of $10 \mathrm{~nm}$ diameter in the center of the nucleus. The periphery containing the thicker fibers is more electron dense than the center.

Stage 4 is at the beginning of nuclear elongation. The nucleus is oval with a short axis of about $3 \mu \mathrm{m}$ diameter, and is filled by wavy $10 \mathrm{~nm}$-chromatin fibers (Fig. 4). Its electron density is less than at stages 1 and 2. In this stage, the acrosomal vesicle adhering to the anterior of the nucleus changes from a round to conical shape. Microtubules develop around the nucleus and extend posteriorly from the base of the acrosome.

In stage 5, the nucleus is more elongated and becomes spindle-shaped and about $2.5 \mu \mathrm{m}$ in width (Fig. 5). Wavy chromatin fibers of $10 \mathrm{~nm}$ width, as in stage 4 , are evenly distributed in the nucleus, and the electron density of the nucleus is similar to that in stage 4 . However, only the chromatin fibers just beneath the nuclear envelope are electron dense and appear as a row of dots (Fig. 5, arrows).

In stage 6 , the nucleus becomes more elongated with a diameter of about $1.0 \mu \mathrm{m}$ (Figs. 6a and b). In a longitudinal section of the nucleus (Fig. 6a) straight chromatin fibers of about $30 \mathrm{~nm}$ width are seen in the periphery of the nucleus (Fig. $6 \mathrm{a}$, arrows). These fibers are very electron dense and are aligned parallel to the nuclear envelope along the long axis of the elongated nucleus. In a cross section of the nucleus in this stage, the fibers are seen as dots arranged in a row along the nuclear envelope. Here and there, they adher to each other (Fig. 6b, double arrows) and in some parts they appear as sheets (Fig. 6b, thick arrows), presumably due the their lateral fusion. In contrast, the chromain fibers in the center of the nucleus are about $10 \mathrm{~nm}$ wide are wavy and entangled with each other. They are less electron dense than those at the periphery.

In stage 7 the nucleus becomes more elongated with a diameter of about 0.8 $1.0 \mu \mathrm{m}$. Chromatin fibers are seen throughout the nucleus. They are all $25-30 \mathrm{~nm}$ in diameter and are straight. In a longitudinal section (Fig. 7a), the few chromatin fibers seen in the periphery of the nucleus appear straight electron dense and arranged in parallel along the long axis of the nucleus (Fig. 7a, arrows). Chromatin fibers in the center of the nucleus are still somewhat wavy, but most of them are alinged along the long axis. The wavy chromatin fibers are less electron dense than the straight ones in the periphery. In cross section (Fig. $7 \mathrm{~b}$ ), the nucleus is not round, but has two shallow dents. Microtubules are seen in a layer just outside the nucleus (Fig. 7b, arrow heads). In the nucleus, chromatin fibers of $30 \mathrm{~nm}$ diameter are seen as dots in cross section, but the chromatin seen just beneath the nuclear envelope appears as a sheet of about $30 \mathrm{~nm}$ thickness (Fig. 7b, arrows). Chromatin fibers appearing as dots and representing the inner portion of the sheet are seen in a row. Some of them are confusing in side by side (Fig. $7 \mathrm{~b}$, double arrow). From the longitudinal and cross sections of the nucleus in stages 6 and 7 , the sheet of chromatin seems to be formed by side-to-side fusion of chromatin fibers of $30 \mathrm{~nm}$ diameter. The fusion of chromatin fibers into a sheet may begin in the periphery of the nucleus and then gradually extend to the center of the nucleus. Then, the sheet of chromatin seems to become a network by adhesion of different part of the sheet, as seen in the cross section of the nucleus in Fig. 8b (stage 8). In 


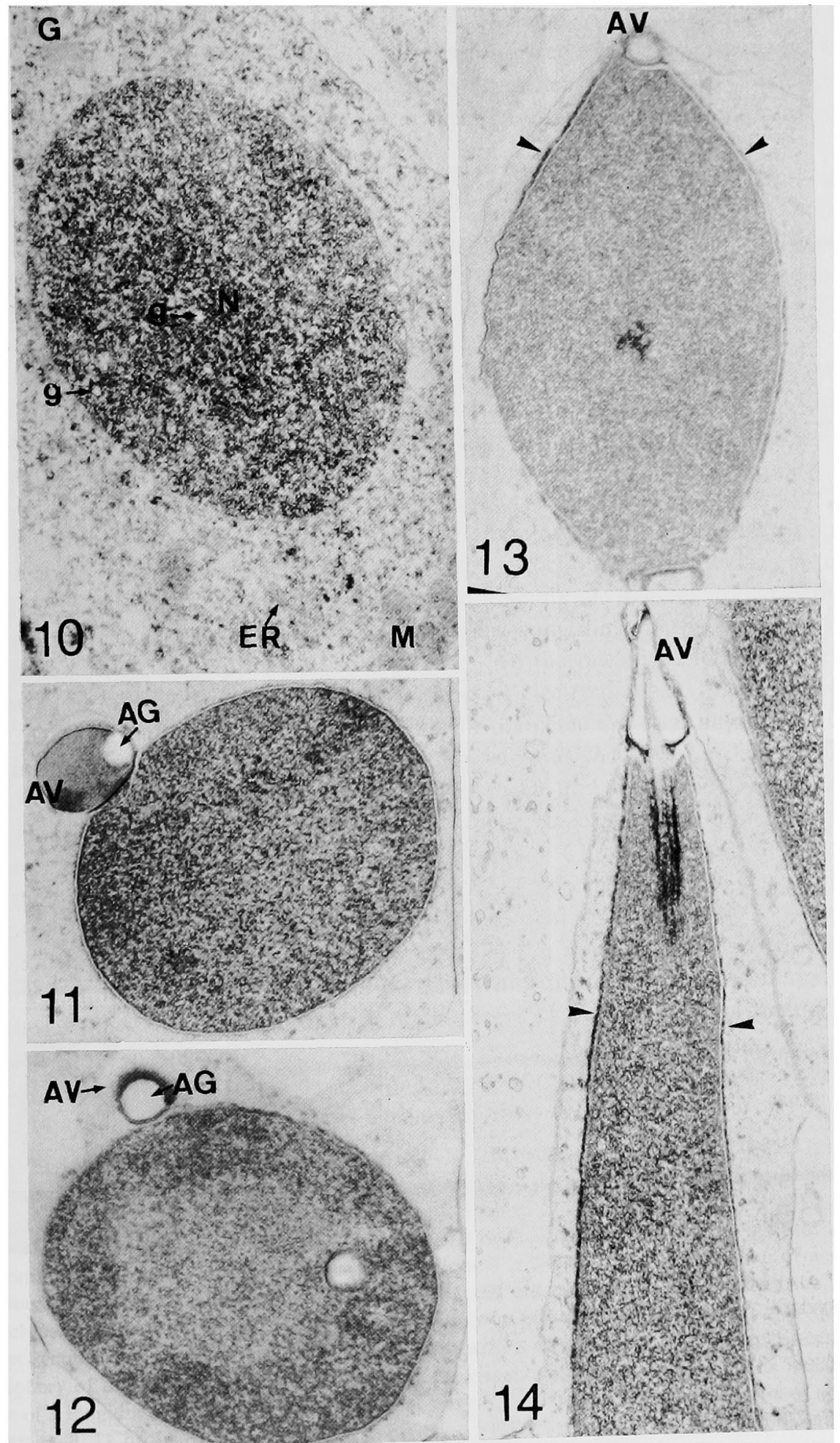



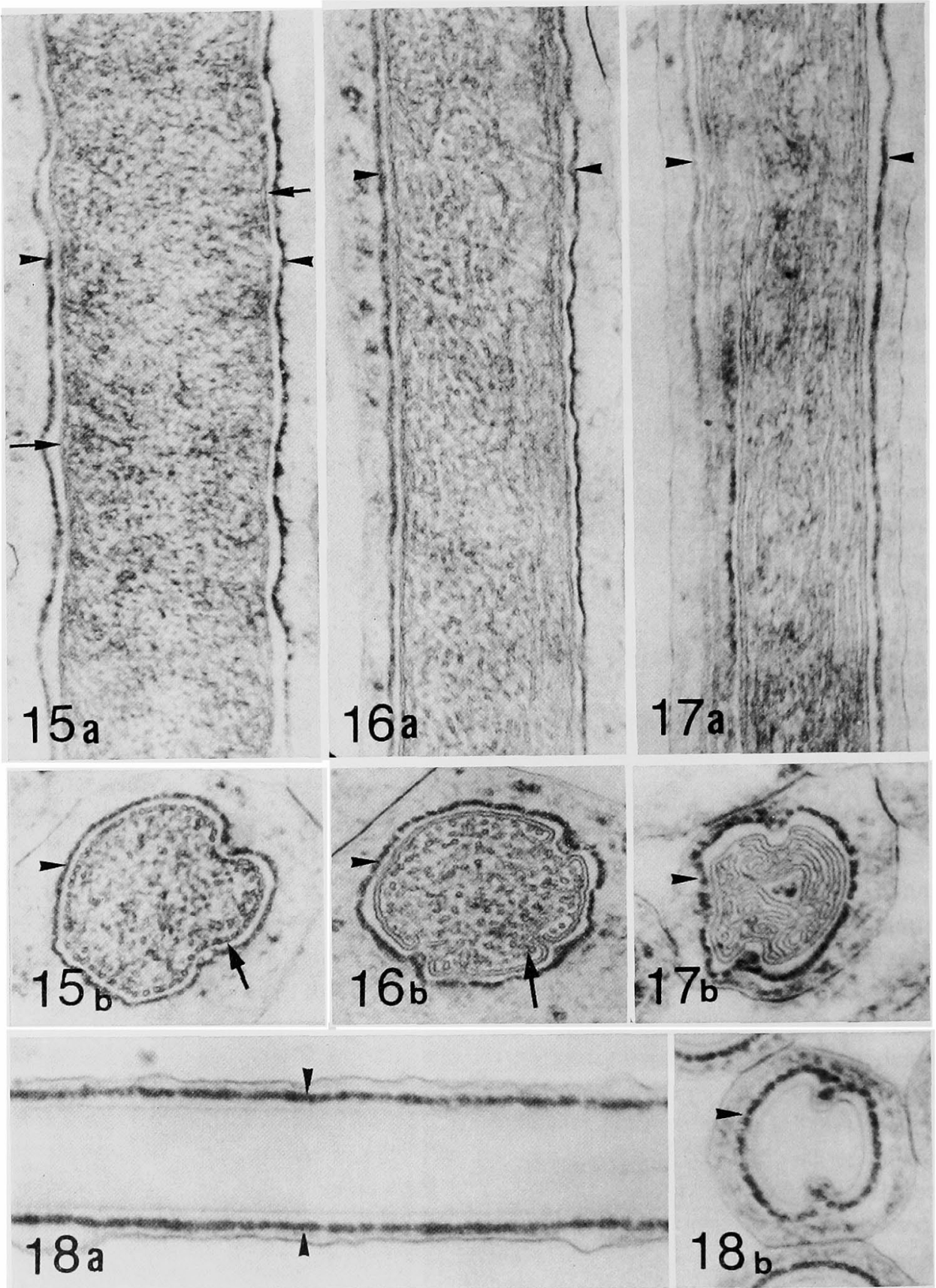
this figure, the nucleus is about $0.5-0.6 \mu \mathrm{m}$ in diameter. In a longitudinal section of the nucleus in this stage (Fig. 8a), chromatin is seen as electron-dense strips, arranged parallel to the long axis of the elongated nucleus. However, judging from cross sections (Fig. 8b), these strips may be profiles of a sheet of chromatin of about $30 \mathrm{~nm}$ thickness.

Stage 9 is the final stage of spermiogenesis in which sperm maturation is complete. In this stage, the nucleus is less than $0.3 \mu \mathrm{m}$ in width. Its interior appears homogeneous in electron opacity without any recognizable profiles of fibers or sheets of chromatin (Figs. 9a and b), although somewhat electron high opaque and amorphous structures are seen.

Figs. 10-18 were obtained by PTA-staining.

According to Courtens and Loir (1981a), PTA stains lysine, but not arginine in mature sperm nuclei, electron microscopically. For determination of whether DNA in spermatozoan nuclei combines with histone or protamine, materials were stained with a solution of PTA in absolute acetone.

Figs. 10-18 show the appearance in stages $1-9$, respectively. Fig. 10 shows a cell in stage 1. The PTA-staining method gives lower contrast than the conventional fixation method. The nuclear envelope, mitochondrial membrane, cisternae of Golgi bodies, and endoplasmic reticulum, all membrane systems in the spermatid cell appear electron transparent. Microtubules consisting of the sperm tail and encircling sperm head appear electron dense. In the nucleus, entangled electrondense chromatin fibers of $10-25 \mathrm{~nm}$ width are electron dense. A few electron transparent granules of about $100-200 \mathrm{~nm}$ in diameter are seen among the chromatin fibers (Fig. 10, g). These may be the same as the electron dense granules seen by conventional fixation, judging from their size and distribution in the nucleus. Fig. 11 shows a nucleus considered to be at the stage just after adhesion of the acrosomal vesicle to the nuclear envelope (stage 2). Chromatin fibers of 10-25 nm width are evenly distributed in the nucleus. Acrosomal granule, including the acrosomal vesicle (Fig. 11, AG) is electron transparent, unlike with the conventional fixation (Fig. 2, AG). The nucleus in Fig. 12 is in stage 3. Chromatin fibers of about $25 \mathrm{~nm}$ width are seen in the periphery and fibers with about $10 \mathrm{~nm}$ width in the center. The electron density of the fibers in the periphery is greater than that of those in the center.

The oval nucleus (stage 4) in Fig. 13 and more elongated nucleus (stage 5) in Fig. 14 are filled evenly with wavy chromatin fibers of $10 \mathrm{~nm}$ diameter. The chro-

Figs. 10-18 were obtained by PTA-staining. 10-14:10, necleus at stage $1 . \times 15000.11$, nucleus at stage $2 . \times 15000.12$, nucleus at stage $3 . \times 15000.13$, nucleus at stage $4 . \times 15000.14$, anterior part of a nucleus at stage $5 . \times 15000$.

Figs. 15-18. 15a, part of a longitudinal section of a nucleus at stage 6 . Arrows indicate straight chromatin of $30 \mathrm{~nm}$ width appearing as three stripes. $\times 30000$. $15 \mathrm{~b}$, cross section of a nucleus at stage 6. Chromatin fibers are circular (arrow) and are arranged in a row along the nuclear envelope. $\times 30000$. 16a, part of a longitudinal section of a nucleus at stage $7 . \times 30000.16 \mathrm{~b}$, cross section of a nucleus at stage 7. Chromatin with a three-layered appearance is seen in the periphery of the nucleus. The arrow indicates the circular profile of chromatin. $\times 30000.17 \mathrm{a}$, part of a longiducudinal section of a nucleus at stage $8 . \quad \times 40000 . \quad 17 \mathrm{~b}$, cross section of a nucleus at stage 8 . Chromatin sheets form a network. $\times 42000$. 18a and $b$, longitudinal and cross sections of nuclei at stage $9 . \times 60000$. 
matin fibers in nuclei in stages 1-5 are all wavy, irrespective of whether they are thick or thin and of high or low electron density. In a more advanced stage of spermiogenesis (stage 6), the nuclei are more elongated and thinner. The nucleus in Fig. 15a is filled with wavy chromatin fibers of about $10 \mathrm{~nm}$ width, as in previous stages. However, chromatin fibers just beneath the nuclear envelope (although the nuclear envelope is not clear with PTA-staining) are straight (Fig. 15a, arrows) and lie along the long axis of the elongated nucleus. These chromatin fibers are about $30 \mathrm{~nm}$ in width and in profile give a pattern of three stripes, dark ( $7 \mathrm{~nm}$ thick), pale (15 nm thick) and dark ( $7 \mathrm{~nm}$ thick). The electron density of these chromatin fibers with a three-striped pattern is less than that of the interior. In cross section of a nucleus at this stage (Fig. 15b), $30 \mathrm{~nm}$-chromatin fibers just beneath the nuclear envelope appear circular with electron dense wall of $7 \mathrm{~nm}$ thickness and are seen arrange in a row adhering to each other (Fig. 15b, arrow).

A nucleus in a more advanced stage of spermiogenesis than that in Fig. 15, is shown in Fig. 16 (stage 7). It is more elongated and thinner (about $0.8 \mu \mathrm{m}$ wide) than in previous stages. In a longitudinal section of the nucleus (Fig. 16a), the wavy fibers seen in the stages 1-6 are not recognized. All the fibers are about $30 \mathrm{~nm}$ in wide and have the same three striped pattern as in the periphery of the nucleus in the previous stage (Fig. 15a, arrows). Most of the chromatin fibers are arranged along the long axis of the nucleus, although there are a few irregularly arranged chromatin fibers in the center. Fig. $16 \mathrm{~b}$ shows a cross-section of a nucleus in the same stage as that in Fig. 16a. This photograph should be compared with Fig. 7b obtained by conventional fixation. Electron dense microtubules are seen around the nucleus (Fig. 16b, arrow heads). The nuclear envelope was too electron transparent to be recognized, but the chromatin just beneath the nuclear envelope shows a three layered pattern of dark, pale and dark stripes of $7 \mathrm{~nm}, 15 \mathrm{~nm}$ and $7 \mathrm{~nm}$ thickness, respectively. This chromatin may correspond to the $30 \mathrm{~nm}$-sheet of chromatin in Fig. $7 \mathrm{~b}$ (arrows). The chromatin fibers in the interior of the nucleus have various profiles: some are circular and about $30 \mathrm{~nm}$ in diameter with an electron dense wall of $7 \mathrm{~nm}$ thickness and are filled with somewhat less electron dense material (Fig. 16b, arrow). Judging from the appearance in longitudinal (Figs. 15a and 16a) and cross (Figs. 15b and 16b) sections of the nucleus in stages 6 and 7, the chromatin of $30 \mathrm{~nm}$ diameter stained with PTA is not in the form of fibers but of tubules. In Figs. $15 \mathrm{~b}$ and $16 \mathrm{~b}$, the tubular chromatin fibers are seen in a row in many places. Some of them adhere to each other side-to-side and give impression of transitional form to three-layered chromatin sheet. The three-layered chromatin sheet seen just beneath the nuclear envelope (Fig. 16b) may be formed by side-to-side fusion of a row of tubular chromatin fibers.

In stage 8 the nucleus is more elongate than in stage 7 . In a longitudinal section of the nucleus (Fig. 17a), all the chromatin shows a three-striped pattern and is arranged in parallel along the long axis of the nucleus. In a corss section of the nucleus (Fig. 17b) at the same stage as that in Fig. 17a, all the chromatin appears as three-layered sheets fused in some places to give the appearance of a maze.

Longitudinal and cross sections of the nucleus in mature spermatozoa in stage 9 are shown in Figs. 18a and b, respectively. No fibers, tubules or sheets of chro- 
matin can be recognized, and the inside of the nucleus has a homogeneous appearance of low electron density, unlike with conventional fixation (Fig. 9).

Comparison of figures of stages 1-5 obtained by the two methods, conventional fxation and PTA-staining, shows that chromatin fibers decrease from $25 \mathrm{~nm}$ (stages $1-3$ ) to $10 \mathrm{~nm}$ (steges 4-5) in diameter during spermiogenesis and that transformation proceeds from the center to the periphery of the nucleus. In contrast, figures of nuclei in stages 6-7 obtained by the two methods (Figs. 6-7 and Figs. 15-16) show that chromatin fibers gradually become thicker, from about $10 \mathrm{~nm}$ to $25-30 \mathrm{~nm}$ in diameter in these stages. At the same time, the chromatin fibers change from a wavy to a straight. Then the chromatin fibers become arranged in parallel along the long axis of the elongated nucleus and fuse side-to-side forming a sheet (Figs. 7-8, and Figs. 16-17). Then the chromatin sheets fuse in various places. These changes of chromatin fibers start at the periphery of the nucleus and proceed toward the center. When fusion is complete in mature spermatozoa of $B$. germanica, the nucleus appears homogeneous in electron density.

\section{Discussion}

In the present studies, morphological alternations of chromatin fibers of $30 \mathrm{~nm}$ diameter during spermiogenesis were followed by conventional fxation and by examining cytochemical alternations detected by the PTA-staining method. The results showed the morphological changes during replacement of basic nuclear proteins.

In substitution of sperm-specific arginine-rich histone or protamine for somatic histone in chromatin fibers, the following process may occur. First, somatic histone must leave the DNA molecules. For this, somatic chromatin fibers must be uncoiled into fundamental filaments, if the chromatin fibers have a solenoid structure formed by coiling of fundamental filament with beads-like structure of about $10 \mathrm{~nm}$ width, as proposed by Finch and Klug (1976). Then, after detachment of somatic histone from DNA molecules, recombination of protamine or sperm-specific arginine-rich histone with the DNA molecules may occur. Sperm-specific chromatin fibers may then be formed by recoiling of the recombine nuclear filaments. Consistent with this possibility, the present observations showed the following processes in transformation of chromatin fibers during spermiogenesis: chromatin fibers in the nucleus early in spermiogenesis (stages 1-3) were wavy and about $25 \mathrm{~nm}$ in width. During spermiogenesis (stages $4-5$ ), they became only about $10 \mathrm{~nm}$ in width and then returned to about $30 \mathrm{~nm}$ in width (stages 6-7). However, these newly formed chromatin fibers (ex. Fig. 7a, arrow) differed from those in earlier stages, as seen in Figs. 1-3, in being straight not wavy. Moreover, the chromatin fibers in intermediate stages (stages 4-5), between stages 1-3 and stages 6-7, were about $10 \mathrm{~nm}$ in width, which is consistent with the fundamental size of filaments in $30 \mathrm{~nm}$ chromatin fibers with a solenoid structure. These findings support the process described above.

With the PTA-staining method, wavy $25 \mathrm{~nm}$-chromatin fibers in the nucleus in stages 1-3 were strongly and completely stained but the chromatin fibers of $10 \mathrm{~nm}$ 
width in the nucleus in stages $4-5$ were stained less. The chromatin fibers of $30 \mathrm{~nm}$ width seen in the nucleus in stages 6-7 were straight, as on conventional fixation, but they stained only at their periphery and appeared tubular (Figs. $15 \mathrm{~b}$ and $16 \mathrm{~b}$, arrows) of which the structure has never been seen by ultrathin sections. If only lysine is stained by PTA as reported by Courtens and Loir (1981a), this difference in stainability on chromatin fibers in early, middle and later stages in spermiogenesis suggests that nuclear basic protein in the chromatin fibers changes during these stages. Moreover, the facts that chromatin fibers in the nucleus in intermediate stages (stages 4-5) are thinner (10 nm in width) and stain less with PTA than those in early stages may be interpreted as indicating that in these stages chromatin fibers unravel into fundamental filaments and lose basic protein. Thus these facts may also support the above presumptions.

By on the basis of these considerations, the process of substitution of nuclear basic protein during spermiogenesis in $B$. germanica may be concluded to be as follows: chromatin fibers in early stages of spermiogenesis (stages 1-3) are of the somatic type, in which DNA molecules are combined with somatic histone. During spermiogenesis, the chromatin fibers unravel into fundamental filaments (stages 4 5). When the chromatin fibers are thin, somatic histone leaves the DNA double helix, and instead, sperm-specific basic protein or arginine-rich protein combines with the DNA double helix. Then, the chromatin filaments recoil, becoming sperm-specific chromatin fibers of about $30 \mathrm{~nm}$ width with a straight contour.

Kierszenbaum and Tres (1978) described packaging processes of chromatin fibers in cricket spermiogenesis. According to them, packaging in chromatin fibers occurs by a 2-step process; the first step is transformation of chromatin fibers from a particulate or beaded type to smooth fibers of uniform diameter, and the second step involves alignment and packing of chromatin fibers by formation of multiple regularly folded packaging units. In the present study packaging of chromatin fibers was also traceable as three steps. First, replacement of nuclear basic protein by arginine-rich histone or protamine (Figs. 1-6 and Figs. 10-15) accompanied by morphological alteration of the chromatin fiber. Second, arrangement of the chromatin fibers (Figs. 6-7 and Figs. 15-16) and third, fusion of chromatin fibers arranged side-to-side (Figs. 7-8 and Figs. 17-16). After these processes, the chromatin fibers became tightly packaged in the small sperm nucleus (Figs. 9 and 18). The first step in the present study, may correspond to the first step of Kierszenbaum and Tres (1978) but the second and third steps differ from their second step.

Sheridan and Barrnett (1969) first reported that alcoholic PTA stains basic proteins, such as lysine and arginine. Since then, a few studies by the PTA-staining method have been reported (Esponda 1978, Kierszenbaum and Tres 1978, Courtens and Loir 1981a and b, Sakai-Wada 1983). However, as described above, Courtens and Loir (1982a) reported that mature sperm nuclei were not stained with PTA although they contained much arginine. Similar results on cricket sperm were reported by Kierszenbaum and Tres (1978). In the present study, the nucleus of mature spermatozoa of B. germanica was also not stained with PTA. Sheridan and Barrnett (1969) reported that both the lysine and guanidyl groups of arginine can bind with alcoholic PTA, whereas Courtens and Loir (1978a) reported that the 
amino residue of lysine binds with PTA, whereas the guanidyl group of arginine in mature mammalian sperm does not combine with PTA for some unknown reason. Warrant and Kim (1978) and Balhorn (1982) proposed model of nucleoprotamine. In the model of Warrant and Kim (1978), protamine molecules, which are represented as several connected cylinders, wrap around the major groove of the DNA double helix. In the model of Balhorn (1982), protamine molecules are the central polyarginine segment lying half in and half out of the minor groove of the DNA double helix. Irrespective of whether protamine molecules are present in the major or minor groove of the DNA double helix, both models propose that protamine molecules wrap around the groove of the DNA double helix by binding of the remaining unbounded phosphate of the DNA to guanidino groups of arginine (Balhorn 1982). Warrant and Kim (1978) also proposed that protamine molecules consist of four helical segments which have four consecutive arginine residues as side chains and that two of these side chains connect the protamine segment with one DNA double helix across its major groove, while the other two side chains connect the protamine segment with the other DNA double helix across its minor groove. Moreover, some segments of the protamine molecule wrap around the major groove of the DNA double helix and the other segments of the same molecule wrap around the major groove of the other DNA double helix. In either case, heavy condensation of DNA double helices occurs. If the present results obtained by the PTA-staining method are interpreted according to the model of Warrant and Kim (1978), the following possibilities with regard to PTA-staining of arginine in sperm nuclei should be considered: the fact that the chromatin fibers of $30 \mathrm{~nm}$ width after replacement of nuclear basic protein by protamine or arginine-rich protein appeared tubular may be explained by supposing that free guanidino groups of arginine, which are targets for binding of PTA, are present only in the periphery of the fibers. In later stages of spermiogenesis (stages 7-8), chromatin apperars as a 3-layerd sheet of $30 \mathrm{~nm}$ thickness, with dark ( $7 \mathrm{~nm}$ thick), pale $(10 \mathrm{~nm}$ thick) and dark $(7 \mathrm{~nm}$ thick) layers (Figs. 16-17), as the result of side-to-side fusion of parallel arrays of $30 \mathrm{~nm}$ chromatin fibers. This phenomenon may be explained by supposing that free guanidino groups of arginine, located on the surface of $30 \mathrm{~nm}$-chromatin fibers combine with DNA double helices, in adjacent $30 \mathrm{~nm}$-chromatin fibers, and that there may be no binding sites for PTA on these faces. If so the stainability with PTA will be lost gradually with the progress of side-to-side fusion of chromatin fibers. When fusion of chromatin fibers is complete, there may be no free guanidino groups of arginine on the chromatin fibers, and thus mature spermatozoa may not stain with PTA (Fig. 18), although they contain much arginine.

The behavior of arginine during spermiogenesis in $B$. germanica will be described elsewhere.

\section{Abstract}

Spermiogenesis in Blattella germanica was examined by electron microscopy with conventional fixation methods and PTA-staining.

Spermiogenesis was studied morphologically over the peroid of replacement 
of nuclear basic protein of somatic histone by sperm-specific arginine-rich protein. Before this time, $30 \mathrm{~nm}$ chromatin fibers uncoil forming $10 \mathrm{~nm}$ chromatin filaments. After the replacement, the chromatin recoils into $30 \mathrm{~nm}$ chromatin fibers. The recoiled chromatin fibers are straight, unlike those before replacement of basic protein which are wavy. This difference in appearance may be due to the replacement of nuclear basic protein of somatic histone by arginine-rich protein or protamine, and differences in localization of these basic proteins in chromatin fibers.

\section{Aknowledgement}

The authors wish to express their thanks to Emer. Prof. and Dr. M. Shigenaga for valuable advice throughout these investigations and also to thank Dr. A. Tanaka for his offer of materials.

\section{References}

Balhorn, R. 1982. A model for the structure of chromatin in mammalian sperm. J. Cell Biol. 93: 298-305.

Courtens, Jean-Luc and Loir, M. 1981a. Ultrastructural detection of basic nucleoproteins: Alcoholic phosphotungstic acid does not bind to arginine residues. J. Ultrastruct. Res. 74: $322-326$.

— and - 1981b. A cytochemical study of nuclear changes in boar, bull, goat, mouse, rat and stallion spermatids. J. Ultrastruct. Res. 74: 327-336.

Esponda, P. 1978. Cytochemistry of kinetochores under electron microscopy. Exp. Cell Res. 114: $247-252$.

Finch, J. T. and Klug, A. 1976. Solenoidial model for superstructure in chromatin. Proc. Nat. Acad. Sci. USA. 73: 1897-1901.

Kierszenbaum, A. L. and Tres, L. L. 1978. The packing unit: a basic structural feature for the condensation of late cricket spermatid nuclei. J. Cell Sci. 33: 265-283.

Sakai-Wada, A. 1983. Ultrastructural and cytochemical studies on the synaptonemal complex of Tradescantia reflexa. Cytologia 48: 859-867.

- and Kuriyama, K. 1980. Electron microscopic studies on acrosome formation in the cockroach, Blattella germanica. Development, Growth and Differentiation 22: 169-177.

Sheridan, W. F. and Barrnett, R. J. 1969. Cytochemical studies on chromosome ultrastructure. J. Ultrastruct. Res. 27: 216-229.

Warrant, R. W. and Kim, Sung-Hou. 1978. $\alpha$-helix-double helix interaction shown in the structure of a protamine-transfer RNA complex and a nucleoprotamine model. Nature 271: 130-135. 